\title{
Antimicrobial activity and cytocompatibility of silver nanoparticles coated catheters via a biomimetic surface functionalization strategy
}

This article was published in the following Dove Press journal:

International Journal of Nanomedicine

2 December 2015

Number of times this article has been viewed

$\mathrm{Ke} \mathrm{Wu}^{\prime}$

Yun Yang ${ }^{2,3}$

Yanmei Zhang ${ }^{2,3}$

Jiexi Deng'

Changjian $\operatorname{Lin}^{2,3}$

'Department of Cardiology, The Affiliated Dongnan Hospital of Xiamen University, Zhangzhou, ${ }^{2}$ Department of Medical Materials, Beijing Medical Implant Engineering Research Center, Beijing Naton Technology Group, Beijing, ${ }^{3}$ State Key Laboratory of Physical Chemistry of Solid Surfaces, Department of Chemistry, College of Chemistry and Chemical Engineering, Xiamen University, Xiamen, People's Republic of China
Correspondence: Jiexi Deng

Department of Cardiology, The Affiliated Dongnan Hospital of Xiamen University, No. 269, Zhanghuazhong Road,

Zhangzhou 363000, People's Republic of China

Tel +865962975537

Fax +865962975534

Email jxdeng175@।63.com

Changjian Lin

State Key Laboratory of Physical Chemistry of Solid Surfaces, College of Chemistry and Chemical Engineering, Xiamen University, Xiamen 36I005,

People's Republic of China

Tel +86592 2189354

Fax +86592 2186657

Email cjlin@xmu.edu.cn
Abstract: Catheter-related bloodstream infections are a significant problem in the clinic and may result in a serious infection. Here, we developed a facile and green procedure for buildup of silver nanoparticles (AgNPs) on the central venous catheters (CVCs) surface. Inspired by mussel adhesive proteins, dopamine was used to form a thin polydopamine layer and induce AgNPs formation without additional reductants or stabilizers. The chemical and physicochemical properties of AgNPs coated CVCs were characterized by scanning electron microscopy, X-ray photoelectron spectroscopy, Raman spectroscopy, and water contact angle. The Staphylococcus aureus culture experiment was used to study the antibacterial properties. The cytocompatibility was assessed by water soluble tetrazolium salts (WST-1) assay, fluorescence staining, and scanning electron microscopy analysis. The results indicated that the CVCs surface was successfully coated with compact AgNPs. AgNPs were significantly well separated and spherical with a size of 30-50 nm. The density of AgNPs could be modulated by the concentration of silver nitrate solution. The antibacterial activity was dependent on the AgNPs dose. The high dose of AgNPs showed excellent antibacterial activity while associated with increased cytotoxicity. The appropriate density of AgNPs coated CVCs could exhibit improved biocompatibility and maintained evident sterilization effect. It is promising to design mussel-inspired silver releasing CVCs with both significant antimicrobial efficacy and appropriate biological safety.

Keywords: surface modification, central venous catheter, antibacterial activity, biocompatibility

\section{Introduction}

Central venous catheters (CVCs) are commonly used to provide venous access for drug delivery, intravenous fluid administration, monitoring hemodynamics, and nutritional support in critically ill patients, but they also are a major source of hospital acquired infection. ${ }^{1}$ Catheter-related bloodstream infections have been associated with increased patient morbidity and mortality in the clinic. ${ }^{2}$ Generally, bacteria adhere on the surface of CVCs followed by growth under suitable environmental conditions to form biofilms, which is elemental to the onset of pathogenesis. To prevent catheter colonization and catheter-related bloodstream infections, a great deal of approaches have been developed in recent years. Current preventive strategies to decrease the risk of these serious infections include the use of antimicrobial agent impregnated catheters and antimicrobial lock therapy. ${ }^{3}$ For example, silver-impregnated CVCs and antiseptic CVCs coated with chlorhexidine and silver sulfadiazine have already been used in clinic medicine. ${ }^{4}$ Silver is widely exploited because of its good antimicrobial action to a wide range of microorganisms. ${ }^{5,6}$ In the meantime, silver nanoparticles (AgNPs), as 
a novel form, are applied in a number of products such as medical device and implant coatings. The catheters can be coated internally and externally with silver containing compounds or impregnated with silver. ${ }^{3}$ Various methods have been used for the synthesis of silver-impregnated CVCs, such as silver iontophoretic technology, solvent casting, electrospinning and electrospraying, plasma based deposition processes, in situ light initiated synthesis, ${ }^{7-10}$ etc.

Recently, mussel-inspired green synthesis of AgNPs on the surface of various materials has drawn a great attention. It has been found that dopamine, could be auto-oxidized to form adhesive polydopamine films onto a wide range of inorganic and organic materials, including classically adhesion-resistant materials, such as polytetrafluoroethylene (PTFE). ${ }^{11,12}$ Many researches reveal that the catechol groups in the polydopamine films could in situ generate the AgNPs without using surfactant or reducing agents. ${ }^{13-18}$ This method has been successfully used for surface modification on various substrate materials, such as microspheres, ${ }^{13}$ fibers, ${ }^{14}$ and polymer films. ${ }^{15,16}$ The polydopamine films also owned strong metal binding ability and adhering capacity to AgNPs. $\mathrm{Xu}$ et al reported that polydopamine modified cotton fabrics with AgNPs showed a higher antibacterial activity even after 30 times washes. ${ }^{17}$ Sureshkumar et al prepared a multilayer of metal nanoparticles on polymer film and found that the surface density of metal nanoparticles could be adjusted by varying the polydopamine coating time. ${ }^{18}$

The commonly used CVCs in the clinic include polyurethane, silicone, and PTFE CVCs. ${ }^{4}$ However, the adhesion force of coatings was rather weak owing to the hydrophobic property of the CVCs, and it was difficult to prepare coatings with good adherence on CVCs. In this study, a mild method for preparing AgNPs/polydopamine/CVCs composites with the AgNPs gradients was presented. The dopamine selfpolymerization was used to form surface-adherent films on the PTFE, CVCs and then the polydopamine was applied to produce uniformly AgNPs. The relationship of the concentration of silver nitrate solution and the density of AgNPs was investigated. Antibacterial activity was investigated by zones of inhibition (ZoI) assay and fluorescence microscopy using Staphylococcus aureus. The toxicity of the AgNPs/ polydopamine/CVCs composites was evaluated by WST-1 and cell adhesion assay.

\section{Materials and methods Materials}

Commercial CVCs (Scw Medicath Ltd, Shenzhen, People's Republic of China) were cleaned by ultrasound in anhydrous ethanol and deionized (DI) water successively before use.
Dopamine hydrochloride was purchased from Sigma-Aldrich, St Louis, MO, USA. Tris(hydroxymethyl)-amino-methane (Tris), silver nitrate, and hydrochloric acid were obtained from Beijing Chemical Plant, Beijing, People's Republic of China. All commercially available reagents and solvents were used without further purification.

\section{Preparation of polydopamine-AgNPs membrane on the catheters}

The CVCs were treated with a dopamine solution $(2 \mathrm{mg} / \mathrm{mL}$, $10 \mathrm{~mm}$ Tris, $\mathrm{pH} \mathrm{8.5)}$ at room temperature with stirring for 20 hours and subsequently ultrasonic washed with DI water, and dried at $60^{\circ} \mathrm{C}$ for 1 hour. The catheters treated with dopamine solution were denoted as DCVC in the subsequent discussion.

The DCVC samples were then immersed in silver nitrate solutions with three different concentrations (1.0, 0.1, and $0.01 \mathrm{M}$ ) for 4 hours at room temperature. Afterward, the samples were ultrasonic cleaned with DI water for 10 minutes and dried at $40^{\circ} \mathrm{C}$ in a vacuum oven. These samples were denoted as DCVC-Ag1, DCVC-Ag2, and DCVC-Ag3, respectively.

\section{Physicochemical characterization}

The surface topography of the specimens was observed by field emission scanning electron microscopy (FE-SEM, Hitachi S4800, Hitachi, Tokyo, Japan). The chemical composition of the surface layer was analyzed using X-ray photoelectron spectroscopy (XPS, VG, Physical Electrons Quantum 2000 scanning ESCA microprobe, Al Ko radiation). The static contact angles (CAs) were measured with an optical contact angle meter system (OCA-20, Dataphysics, Stuttgart, Germany) at ambient humidity and temperature. The DI water droplets used for the CA measurement were $3 \mu \mathrm{L}$ and the three measurements were performed on each sample for statistical accountability. Raman spectra were recorded using Raman spectroscopy (XploRA, Horiba Jobin Yvon, Longjumeau, France) with a $532 \mathrm{~nm}$ air cooled argon ion laser at wavelengths from 50 to $2,000 \mathrm{~cm}^{-1}$.

\section{Antibacterial assay}

Samples were cut into small disks with a diameter of $6 \mathrm{~mm}$ and sterilized with $75 \%$ alcohol for 1 hour and rinsed with phosphate-buffered saline (PBS) for three times. S. aureus strains were grown overnight in Luria-Bertani medium at $37^{\circ} \mathrm{C}$ for 12 hours. The bacterial concentrations were adjusted to $10^{5}$ colony-forming units (CFUs) $/ \mathrm{mL}$ in the antibacterial assay and dispersed uniformly on the surface of Luria-Bertani plate. The sterile samples were laid on the top of the plate and 
incubated at $37^{\circ} \mathrm{C}$ for 12 hours. Then the diameters of zones of inhibition against $S$. aureus were measured.

For the fluorescent staining assay, bacteria were inoculated on the samples mentioned earlier. The concentration of S. aureus was adjusted to $10^{8} \mathrm{CFU} / \mathrm{mL}$. After co-incubated at $37^{\circ} \mathrm{C}$ for 6 hours, samples were removed and rinsed with PBS, stained using acridine orange for 15 minutes in darkness, and then observed by fluorescence microscopy (Olympus Corporation, Tokyo, Japan).

\section{Biocompatibility assay}

MC3T3-E1 cell (Cell Resource Center, PUMC, Beijing, People's Republic of China) was cultured in $\alpha$-minimum essential medium ( $\alpha$-MEM) containing 10\% fetal bovine serum (FBS) and incubated in a humidified atmosphere of $5 \% \mathrm{CO}_{2}$ at $37^{\circ} \mathrm{C}$. Before cell seeding, all the samples were cut into $8 \mathrm{~mm} \times 8 \mathrm{~mm} \times 1 \mathrm{~mm}$. The sterile samples were placed in the 24-well microplates, and the cells were seeded onto the samples at a density of $1 \times 10^{4}$ cells per well.

The viability of cells on the samples was assessed by WST-1 cell proliferation and cytotoxicity assay kit (Beyotime, Nantong, People's Republic of China). After 1, 3, and 7 days of culture, $50 \mu \mathrm{L}$ WST-1 solution was added to each well and incubated at $37^{\circ} \mathrm{C}$ for 2 hours. After incubation, the absorbance values were measured at $450 \mathrm{~nm}$ with a reference wavelength of $690 \mathrm{~nm}$ (Infinite M200 Pro, Tecan Schweiz AG, Männedorf, Switzerland).

For the fluorescent staining assay, cells were stained with $3 \mu \mathrm{M}$ Calcein-AM (Sigma-Aldrich) after 3-day incubation on the samples, and then observed by fluorescence microscopy (Olympus Corporation). The morphology of MC3T3-E1 cells on the samples was investigated by the SEM analysis. After 3 and 7 days of culture, samples were removed from the medium, washed with PBS, fixed in $2.5 \%$ glutaraldehyde, and dehydrated in series of ethanol concentrations. After the ethanol was substituted by tert-butanol, samples were lyophilized and sputter coated with gold before examination under the SEM analysis.

\section{Statistical analysis}

Triplicate experiments were performed. The data were expressed as the mean \pm standard deviation. Analysis of the results was carried out using Student's $t$-test, with the significance level of $P<0.05$ and highly significance level of $P<0.01$.

\section{Results}

\section{Characterization of AgNPs modified CVC surface}

The SEM images for CVC, DCVC, and DCVC-Ag samples are shown in Figure 1. The pristine CVC exhibited an uneven surface, which was fully covered by polydopamine after impregnation in dopamine solution for 24 hours. After immersing into different concentrations of silver nitrate solutions, conductive and monodispersed spherical nanoparticles with a size of $30-50 \mathrm{~nm}$ could be found on the DCVC surface, indicating that the polydopamine surface could spontaneously reduce $\mathrm{Ag}^{+}$ions into AgNPs without requiring additional reducing agent. As shown in Figure $1 \mathrm{C}$ and D, DCVC-Ag1 sample has the largest surface density, and the density decreased with silver nitrate solution concentration.

The relative surface wettability was determined by the static water CA. As shown in Table 1, the pristine catheter showed hydrophobic property with the CA value of $113.3^{\circ} \pm 2.2^{\circ}$. After modifying with surface-adherent polydopamine films, the DCVC showed a hydrophilic surface with the CA value of $80.4^{\circ} \pm 1.8^{\circ}$. The following in situ generation of AgNPs on the DCVC surface resulted in slightly improved hydrophilicity. The CA value decreased as the AgNPs number increased, and that of DCVC-Ag1 samples were nearly constant $\left(52.2^{\circ} \pm 2.4^{\circ}\right)$.

The surface chemical compositions of the CVCs films before and after surface modification were determined by XPS. As shown in Figure 2, the sharp peak of F1s developed from pristine CVCs vanished after polydopamine modification. Meanwhile, no peak corresponding to N1s could be observed on the pristine CVCs, which indicated the nitrogen atom was derived from the polydopamine membrane on DCVC. This result suggests that the thickness of the deposited polydopamine layer is higher than the probing depth of the XPS technique (approximately $7.5 \mathrm{~nm}$ in an organic matrix). ${ }^{19}$ The Ag concentrations determined from the XPS survey spectra of DCVC-Ag1, DCVC-Ag2, and DCVC-Ag3 are 9.64, 6.74 and 1.19 at\%, respectively (Table 1). The highresolution XPS spectra of Ag obtained from DCVC-Ag1, DCVC-Ag2, and DCVC-Ag3 are shown in Figure 3. The binding energies of $\mathrm{Ag} 3 \mathrm{~d}$ doublet peaks located at 368.5 and $374.5 \mathrm{eV}$ can be assigned to $\mathrm{Ag} 3 \mathrm{~d}_{5 / 2}$ and $\mathrm{Ag} 3 \mathrm{~d}_{3 / 2}$ of metallic $\mathrm{Ag}^{0}$, indicating that $\mathrm{Ag}$ mainly exists in the $\mathrm{Ag}^{0}$ state on the DCVC surface. ${ }^{20,21}$

Silver is one of the best materials for making surface enhanced Raman scattering active surfaces, and a great deal of effort has been put into perfecting methods of forming silver surfaces for strong surface enhanced Raman scattering signals. ${ }^{22}$ The chemical composition on the surface was further investigated by Raman spectroscopy. As shown in Figure 4, the peak at $1,330 \mathrm{~cm}^{-1}$ (amide III) is assigned to the vibrational mode of dopamine, which is a distortion of the $\mathrm{C}-\mathrm{C}$ bond connecting the two oxygen-bearing carbon atoms, 

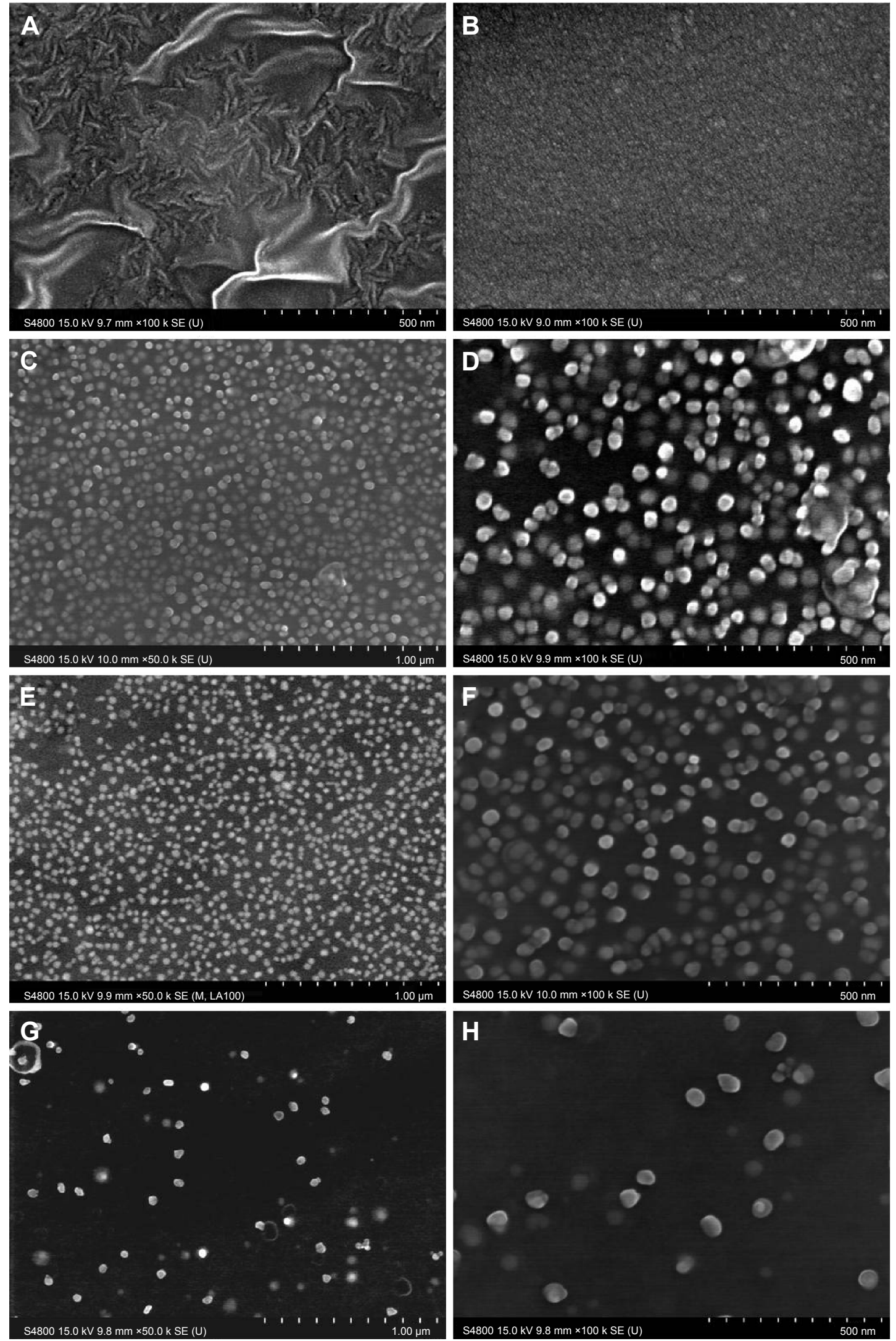

Figure I SEM images of (A) CVC, (B) DCVC, (C and D) DCVC-AgI, (E and F) DCVC-Ag2, and (G and H) DCVC-Ag3.

Abbreviations: CVC, central venous catheter; DCVC, central venous catheters coated with polydopamine films; SEM, scanning electron microscopy 
Table I Surface chemical composition and water contact angle of pristine CVC, DCVC, and DCVC-Ag samples (atomic percentage according to XPS analysis)

\begin{tabular}{|c|c|c|c|c|c|c|c|}
\hline & \multicolumn{6}{|c|}{ Atomic concentration (at\%) } & \multirow[t]{2}{*}{ Contact angle $\left({ }^{\circ}\right)$} \\
\hline & $\mathrm{Cls}$ & NIs & Ols & FIs & Si2p & Ag3d & \\
\hline CVC & 46.21 & - & 8.92 & 37.56 & 7.31 & - & $113.3 \pm 2.2$ \\
\hline DCVC & 61.25 & 5.38 & 23.95 & 0.77 & 8.65 & - & $80.4 \pm 1.8$ \\
\hline DCVC-AgI & 52.43 & 4.20 & 21.87 & 5.60 & 6.26 & 9.64 & $52.2 \pm 2.4$ \\
\hline DCVC-Ag2 & 56.25 & 5.66 & 25.58 & 0.97 & 4.80 & 6.74 & $59.3 \pm 3.9$ \\
\hline DCVC-Ag3 & 55.29 & 5.46 & 27.02 & 1.45 & 9.61 & 1.19 & $66.3 \pm 2.7$ \\
\hline
\end{tabular}

Note: These data is expressed as the mean $\pm S D(n=3)$.

Abbreviations: CVC, central venous catheter; DCVC, central venous catheters coated with polydopamine films; XPS, X-ray photoelectron spectroscopy; SD, standard deviation.

and the peak at 1,595 $\mathrm{cm}^{-1}$ (amide II) is related to in plane $\mathrm{N}-\mathrm{H}$ bending and $\mathrm{C}-\mathrm{N}$ stretching of dopamine, both of which corresponding to characteristic aromatic groups in polydopamine.

The intensity of characteristic peaks of aromatic groups was quite weak in DCVC, and no peaks could be detected in CVC. However, the intensities of the characteristic peaks were obviously increased in the DCVC-Ag samples, and proportional to the density of AgNPs. SEM, XPS, and Raman spectra provide unequivocal proofs that metallic AgNPs have been successfully coated on the CVCs surface.

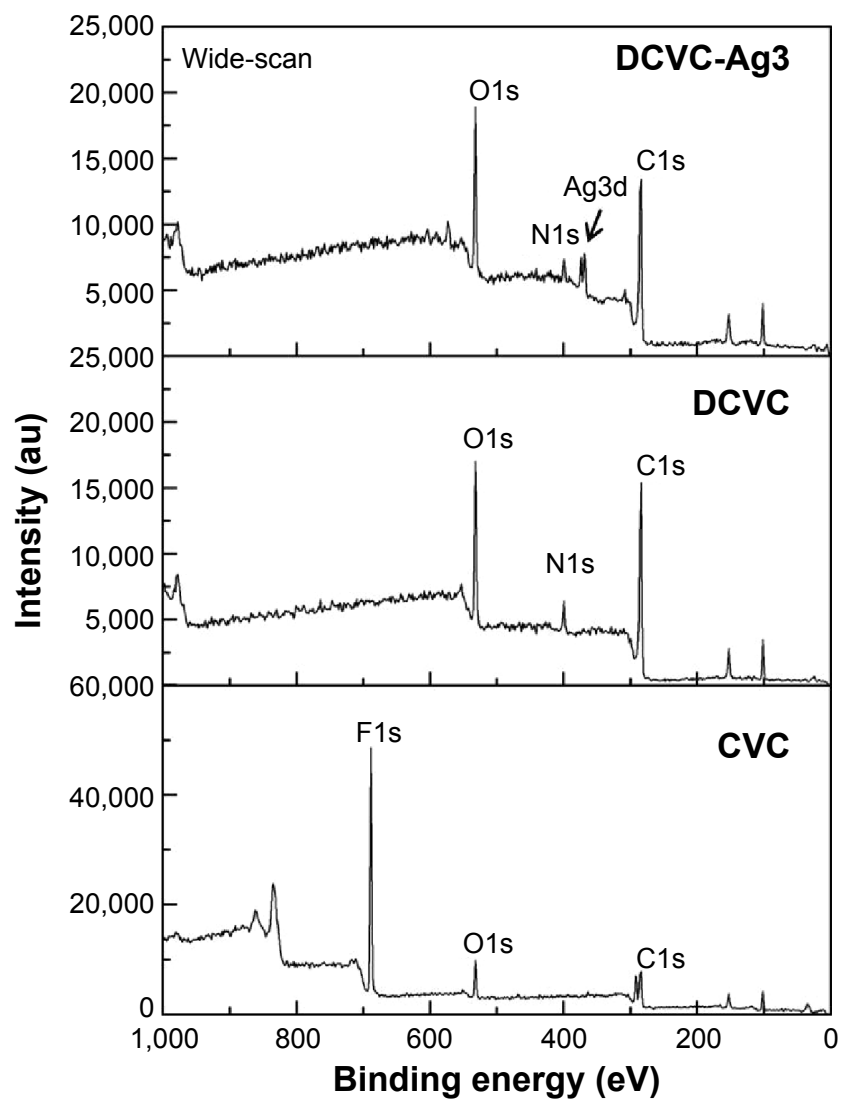

Figure 2 The survey XPS spectra of CVC, DCVC, and DCVC-Ag3. Abbreviations: au, arbitrary unit; CVC, central venous catheter; DCVC, central venous catheters coated with polydopamine films; XPS, X-ray photoelectron spectroscopy.

\section{Antibacterial characteristics of the silvered CVC films}

The ability of the DCVC-Ag samples to prevent viable bacteria colonization was verified by Zol test and fluorescence staining. The Zol test is based on the leaching of silver ions from the surface, the inhibition of the bacterial growth depends on a sufficient concentration of silver ions in the surrounding aqueous environment. ${ }^{23}$ Figure 5 shows the Zol test results by DCVC-Ag1, DCVC-Ag2, and DCVC-Ag3 on S. aureus. All the DCVC-Ag samples showed a clear inhibition zone, which indicated the efficient antibacterial ability of these samples. The diameter of the bacteria inhibition zone ranged from 0.2 to $0.35 \mathrm{~cm}$ against $S$. aureus. Based on the Ag concentrations in Table 1, the antibacterial effect could be modulated by the density of coated AgNPs.

The antimicrobial effect of DCVC-Ag samples was also confirmed by bacteria viability after 6 hours of incubation. As shown in Figure 6, large amounts of viable bacteria were observed on the surface of DCVC (Figure 6A). There were a relatively small number of viable bacteria on the DCVC-Ag3 (Figure 6D). Due to the increased Ag concentrations in DCVC$\mathrm{Ag} 2$ and DCVC-Ag1, much fewer bacteria can be detected on DCVC-Ag2 and nearly no viable bacteria on DCVC-Ag1.

\section{Cytocompatibility assays}

The WST-1 assay was used to evaluate the cytotoxicity of the substrates on MC3T3-E1 cells, and the optical density (OD) value was considered to indicate cell growth on different surfaces. As shown in Figure 7, the OD value of both CVC and DCVC increased obviously with the culture time, and the OD value of DCVC was slightly higher than CVC. On day 1 , there was no appreciable difference on the OD value among all the samples $(P>0.05)$. On day 3 , proliferation of MC3T3-E1 cells in DCVC-Ag1 and DCVC-Ag2 group was markedly inhibited as compared to DCVC-Ag3 $(P<0.05)$, 


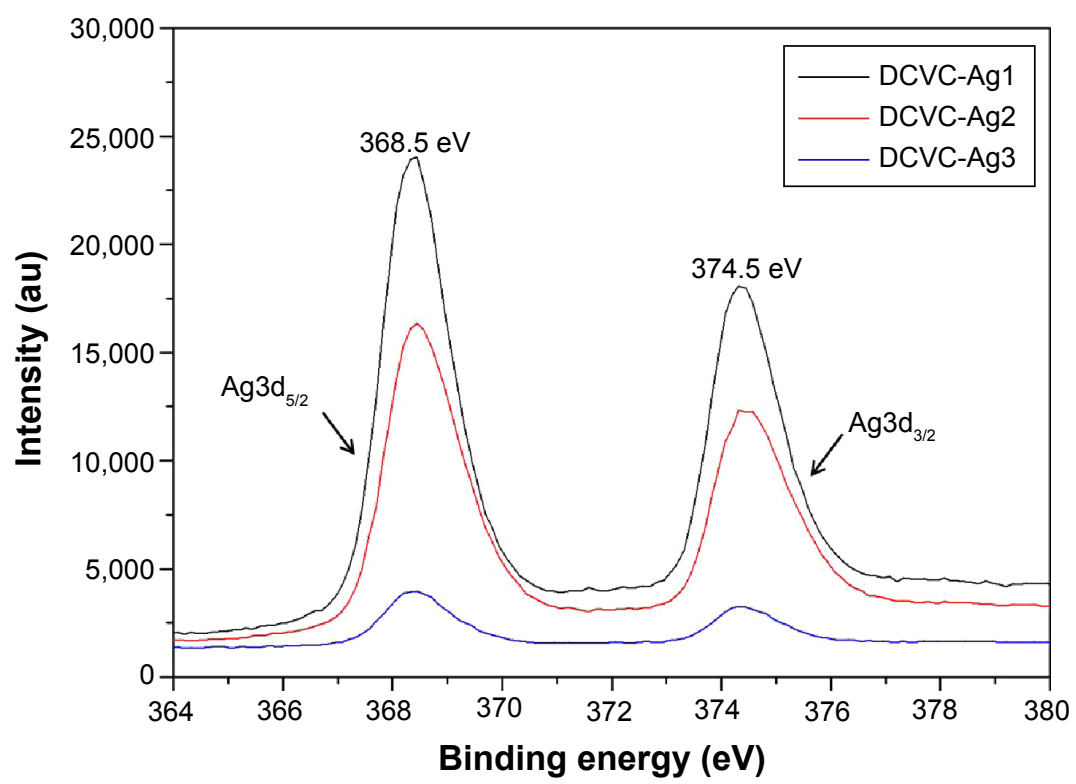

Figure 3 The high-resolution XPS spectra of DCVC-AgI, DCVC-Ag2, and DCVC-Ag3.

Abbreviations: au, arbitrary unit; DCVC, central venous catheters coated with polydopamine films; XPS, X-ray photoelectron spectroscopy.

and the tendencies were even more obvious after 7 days incubation. The cytotoxicity caused by the DCVC-Ag samples is due to the Ag ions released from the coated AgNPs. In the DCVC-Ag3 group, the OD value increased with the culture time. Though the OD values were slightly lower than that of CVC and DCVC, the difference is statistically insignificant. Hence, DCVC-Ag3 exhibited good cytocompatibility compared with DCVC-Ag1 and DCVC-Ag2.
Fluorescence microscopy and SEM were used to characterize adhesion and spreading of cells cultured on different substrates after 3-day incubation. As shown in Figure 8, well adhesion and growth of cells were seen on the surface of DCVC. After co-incubated with different DCVC-Ag sample for 3 days, cells showed an obvious dose-dependent decrease in cell viability compared with that of the DCVC group. Figure 9 shows the cell morphology on different

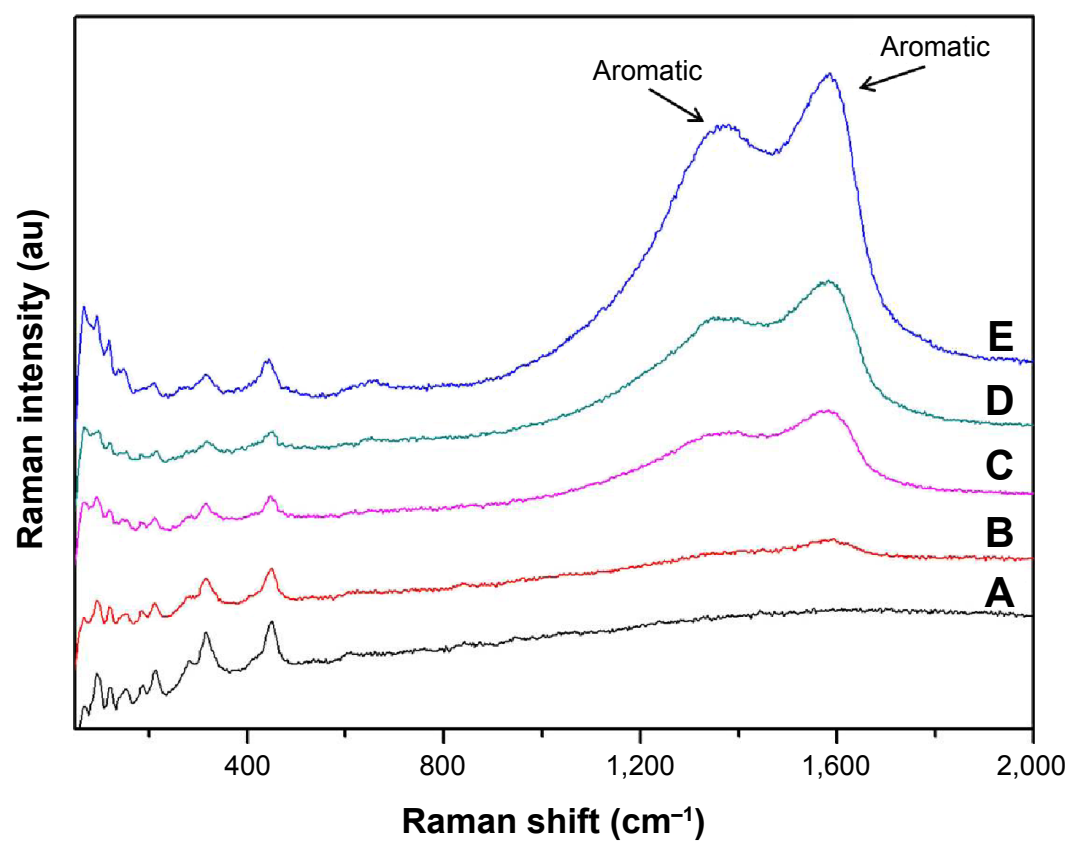

Figure 4 Raman spectra of various samples: (A) CVC, (B) DCVC, (C) DCVC-Ag3, (D) DCVC-Ag2, and (E) DCVC-AgI.

Abbreviations: au, arbitrary unit; CVC, central venous catheter; DCVC, central venous catheters coated with polydopamine films. 




Figure 5 Photographs showing the zone of inhibition assay for DCVC-Ag samples. Abbreviation: DCVC, central venous catheters coated with polydopamine films.

substrates, which assisted to understand the cell behavior. The spread of cells was remarkably regulated by the amount of AgNPs. After 3 days of culture, cells on DCVC and DCVC-Ag3 displayed a polygonal shape with prominent filopodia protrusions. On the contrary, DCVC-Ag1 and DCVC-Ag2 are unfavorable to cell growth. It is noted that the filopodia protrusions vanished on DCVC-Ag1.
After 7 days, a great quantity of cells adhered to the surface of DCVC and DCVC-Ag3, and numerous pseudopodia spread around the cell body. The number of adherence cells on DCVC-Ag1 and DCVC-Ag2 surfaces were significantly less than on DCVC-Ag3, and no filopodia protrusions could be found. The cell morphology again confirmed the best compatibility of DCVC-Ag3. These results were consistent with the WST-1 assay.

\section{Discussion}

Based on the earlier studies, dopamine could self-polymerize to form nanometers thickness of multifunctional adhesive film on various surfaces. It is known that polydopamine can reduce the silver ions to form AgNPs. The catechol groups in the polydopamine film could chelated with $\mathrm{Ag}$, and then in situ reduced the $\mathrm{Ag}^{+}$to $\mathrm{Ag}^{0}$. And the $\mathrm{Ag}^{0}$ could be bonded on the $\mathrm{N}$-site and $\mathrm{O}$-site in polydopamine film and formed seed precursor. The seed $\mathrm{Ag}^{0}$ could grow to nanoparticles through the atom by atom growth with the reduction of $\mathrm{Ag}^{+} \cdot{ }^{17}$ In this study, the particle coverage of the surface increased with silver nitrate solution concentration obviously. The huge difference in the particle coverage of surface is mainly due to the silver precursor concentration.
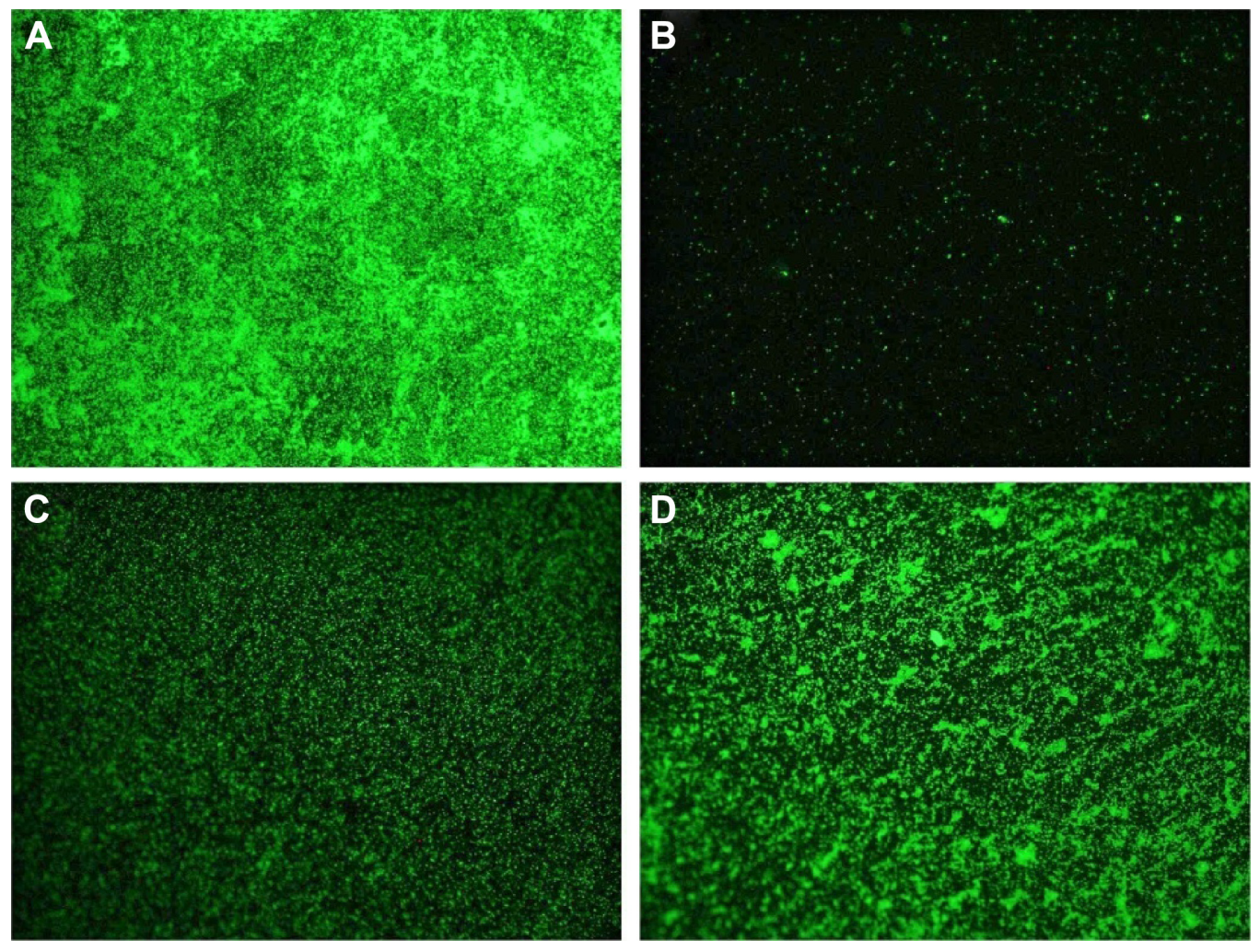

Figure 6 Fluorescent microscope images of Staphylococcus aureus stained on (A) DCVC, (B) DCVC-AgI, (C) DCVC-Ag2, and (D) DCVC-Ag3 after 6 hours of culture. Abbreviation: DCVC, central venous catheters coated with polydopamine films. 


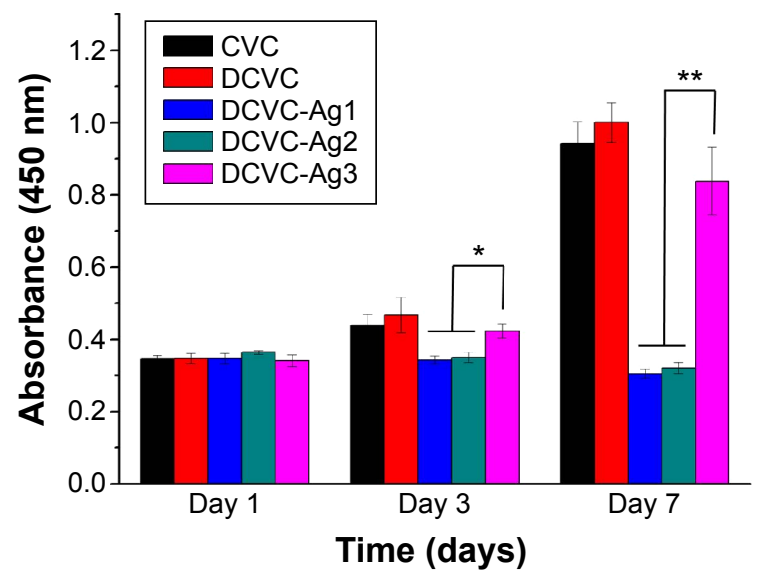

Figure 7 WST-I assay for proliferation of MC3T3-EI cell on different samples at various incubation periods.

Note: Error bars represent mean $\pm S D(n=5), * P<0.05$, ** $P<0.01$.

Abbreviations: CVC, central venous catheter; DCVC, central venous catheters coated with polydopamine films; SD, standard deviation; WST-I, water soluble tetrazolium salts.

As an antibacterial agent, AgNPs coating has been applied in several medical devices, among which catheters, ${ }^{24}$ drains, ${ }^{25}$ and wound dressings ${ }^{26}$ are the most prominent representatives. When it enters the body, metallic silver will react and result in the formation and release of silver ions, which are highly
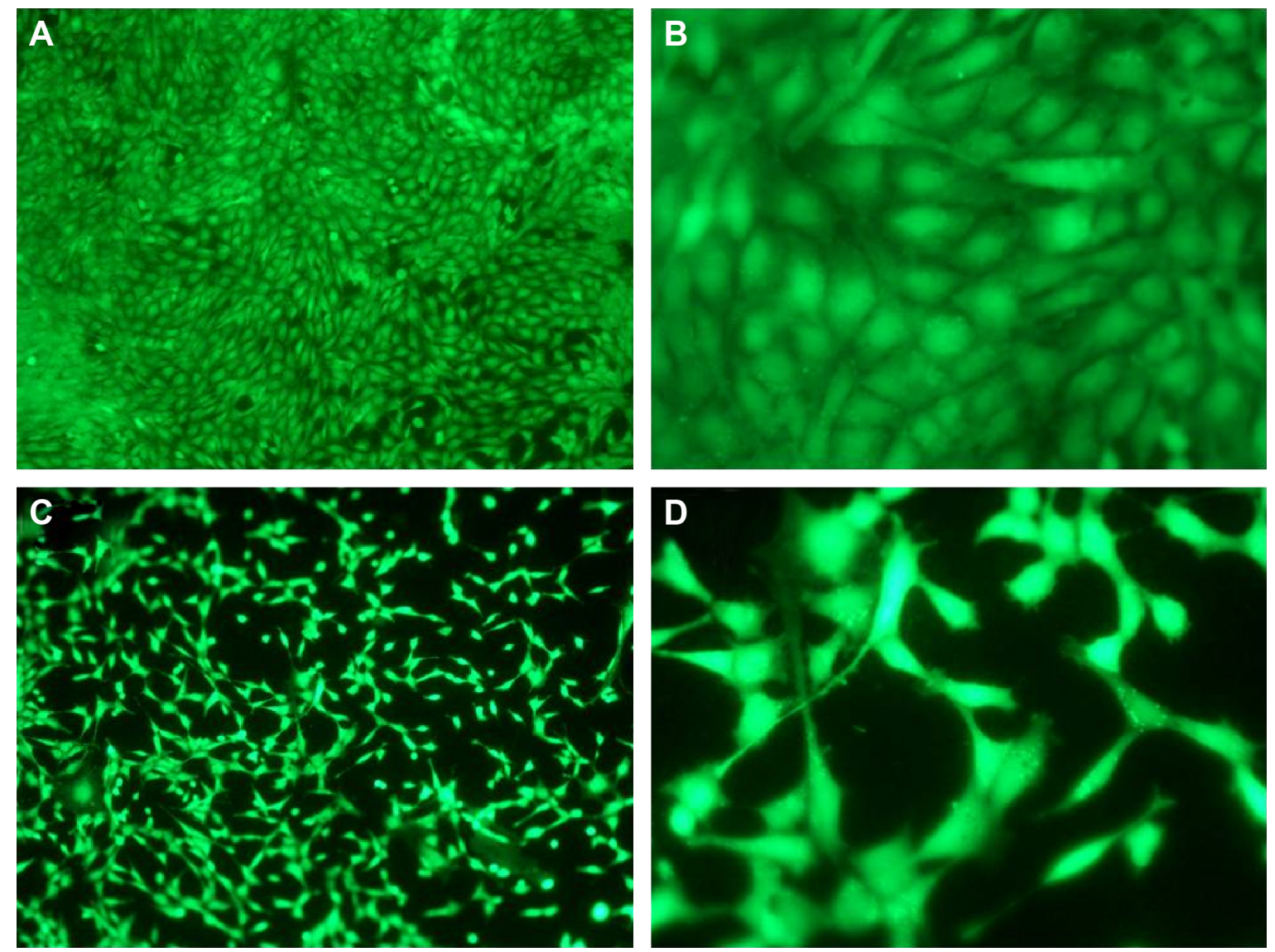

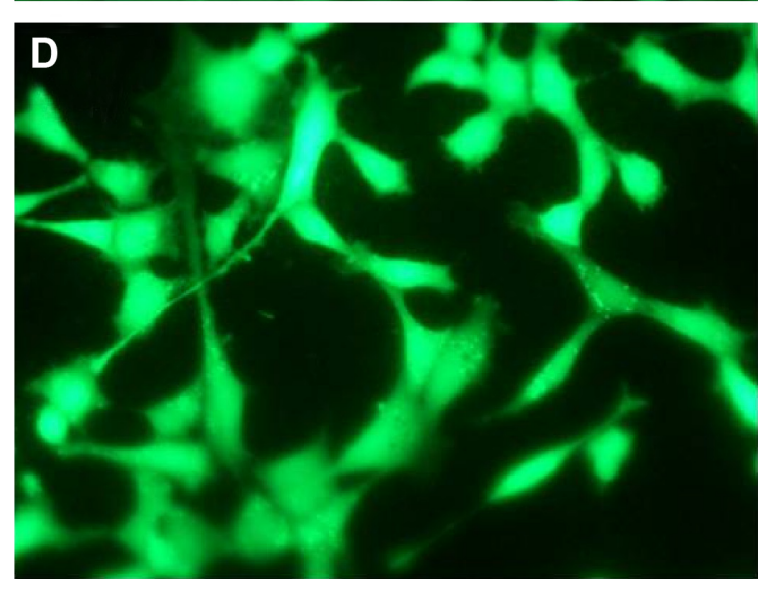

potent as an antibacterial agent. In this study, the antimicrobial effects of AgNPs coated CVCs were investigated. The CVCs coated with spherical AgNPs with a size of 30-50 nm showed strong antimicrobial ability. As shown in Figure 5, all DCVC-Ag samples show obvious antibacterial activity and the killing efficiency is dependent on the AgNPs dose. When incubated with $S$. aureus, the DCVC-Ag samples are highly effective against bacteria and the antibacterial ability is positively correlated with AgNPs density as shown in Figure 6. It is worth mentioning that intensive bacteria $\left(10^{8} \mathrm{CFUs} / \mathrm{mL}\right)$ were used in our antibacterial assay, and the condition is much harsher than the real in vivo environments.

However, one problem of AgNPs is their biotoxicity, thus, AgNPs coatings must be well tolerated. The biosafety of AgNPs has attracted widespread attention for the disappointing results of metallic silver in contact with blood and tissues. ${ }^{6,27}$ Generally, it is accepted that a high dose of silver can induce cytotoxicity. It is also reported that the cytotoxicity of AgNPs can be attributed to continuous leaching of $\mathrm{Ag}^{+}$and its accumulation in the culture medium. ${ }^{28} \mathrm{Ag}^{+}$ release from AgNPs was detected from many aspects (such as size, shape, surface properties, and degree of aggregation),

Figure 8 (Continued) 

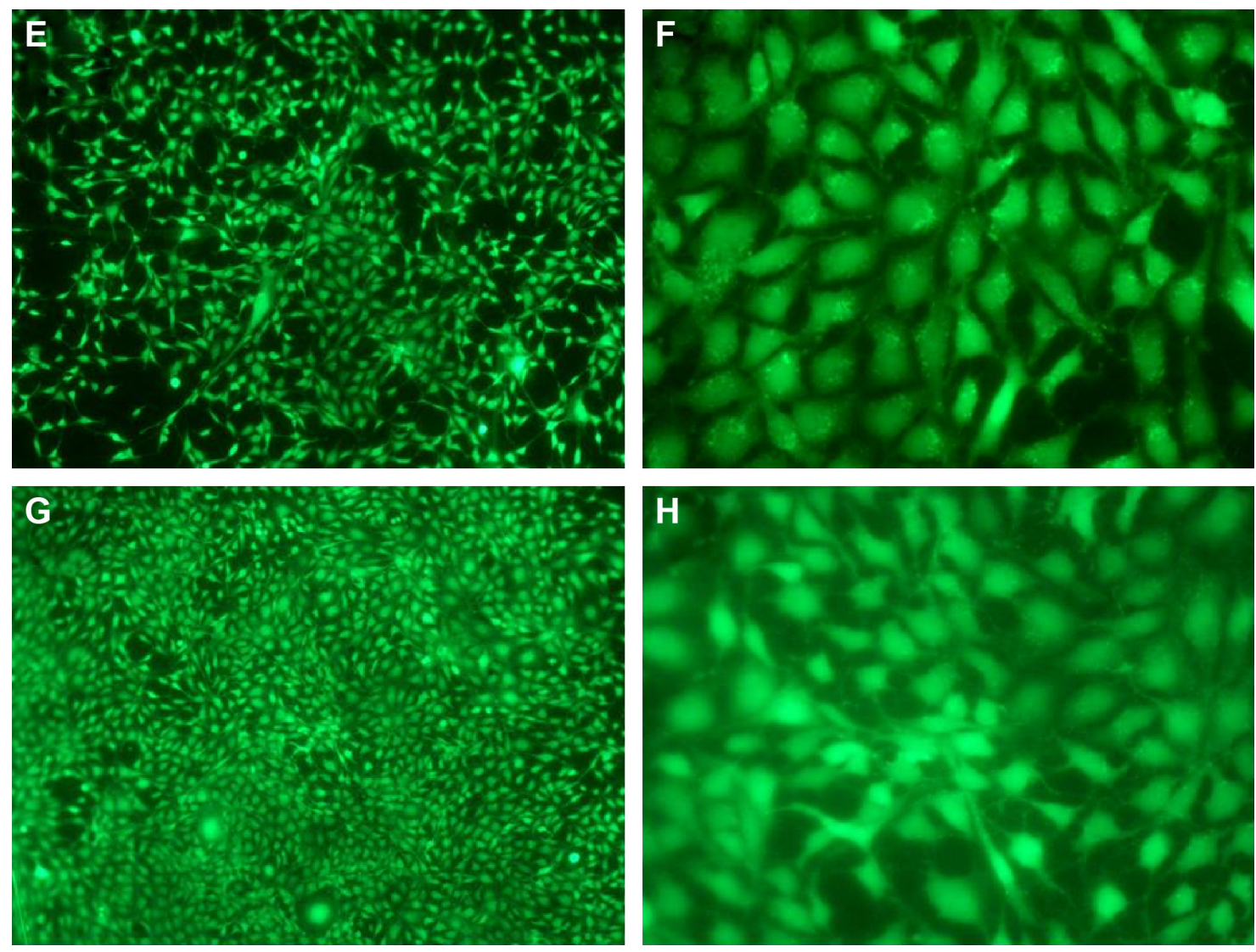

Figure 8 Fluorescence microscopy images of Calcein-AM stained MC3T3-EI cell attached on (A and B) DCVC, (C and D) DCVC-AgI, (E and F) DCVC-Ag2, and (G and H) DCVC-Ag3 for 3 days.

Abbreviation: DCVC, central venous catheters coated with polydopamine films.

especially the particle size..$^{29}$ In light of an earlier report, the cytotoxicity of AgNPs was primarily mediated by a sizedependent release of $\mathrm{Ag}^{+}$, and the smaller AgNPs (with a diameter of $50 \mathrm{~nm}$ ) could facilitate fast dissolution and release of $\mathrm{Ag}^{+} .{ }^{30}$ There is little literature discussed the toxicity of the dopamine reduced AgNPs coatings. Sureshkumar et al showed that the AgNPs deposited on polydopamine coated polyethylene possessed effective biocidal properties and did not inhibit the growth of L-929 cells mouse fibroblasts after 24-hour co-incubation. ${ }^{31}$ But long-term toxicity should be detected for such AgNPs coated materials. It is reported that the biocompatibility and toxicity of AgNPs were highly dependent on the density of AgNPs. ${ }^{32}$ The nature of the silver particles as well as how these are incorporated in the coating will determine the efficacy of such modified medical devices. ${ }^{6}$ In general, it has been suggested that Ag ions are considered to be biocompatible only at relatively lower concentrations. ${ }^{33-35}$ The cytocompatibility property of the AgNPs coated CVCs was also studied. All the DCVC-Ag samples showed no cytotoxicity on day 1 . The results are consistent with that from Sureshkumar et al. ${ }^{31}$ However, obvious cytotoxicity were observed on DCVC-Ag1 and DCVC-Ag2 on day 3 and day 7, which may due to the relative high density of AgNPs. It is possible that the bacteria could be efficiently killed without cell cytotoxicity at specific AgNPs density. DCVC-Ag3 demonstrated both obvious antibacterial property and good biocompatibility in this study. Thus, the CVCs with proper AgNPs coatings via this green preparation method are promising in clinical application. Extended future works including in vivo test are compulsory for these CVCs with AgNPs coatings.

\section{Conclusion}

In summary, we developed a facile biomimetic strategy to decorate uniformly small and well dispersed AgNPs on CVC surface. The SEM results indicated that the particle size was within the range of $30-50 \mathrm{~nm}$, and the content of the nanoparticles could be controlled by the concentration of silver nitrate solution. The XPS and Raman analysis confirmed the presence of metallic silver particles on the CVC films. The AgNPs coated CVC samples showed excellent antibacterial efficacy, and the antibacterial ability was associated with 

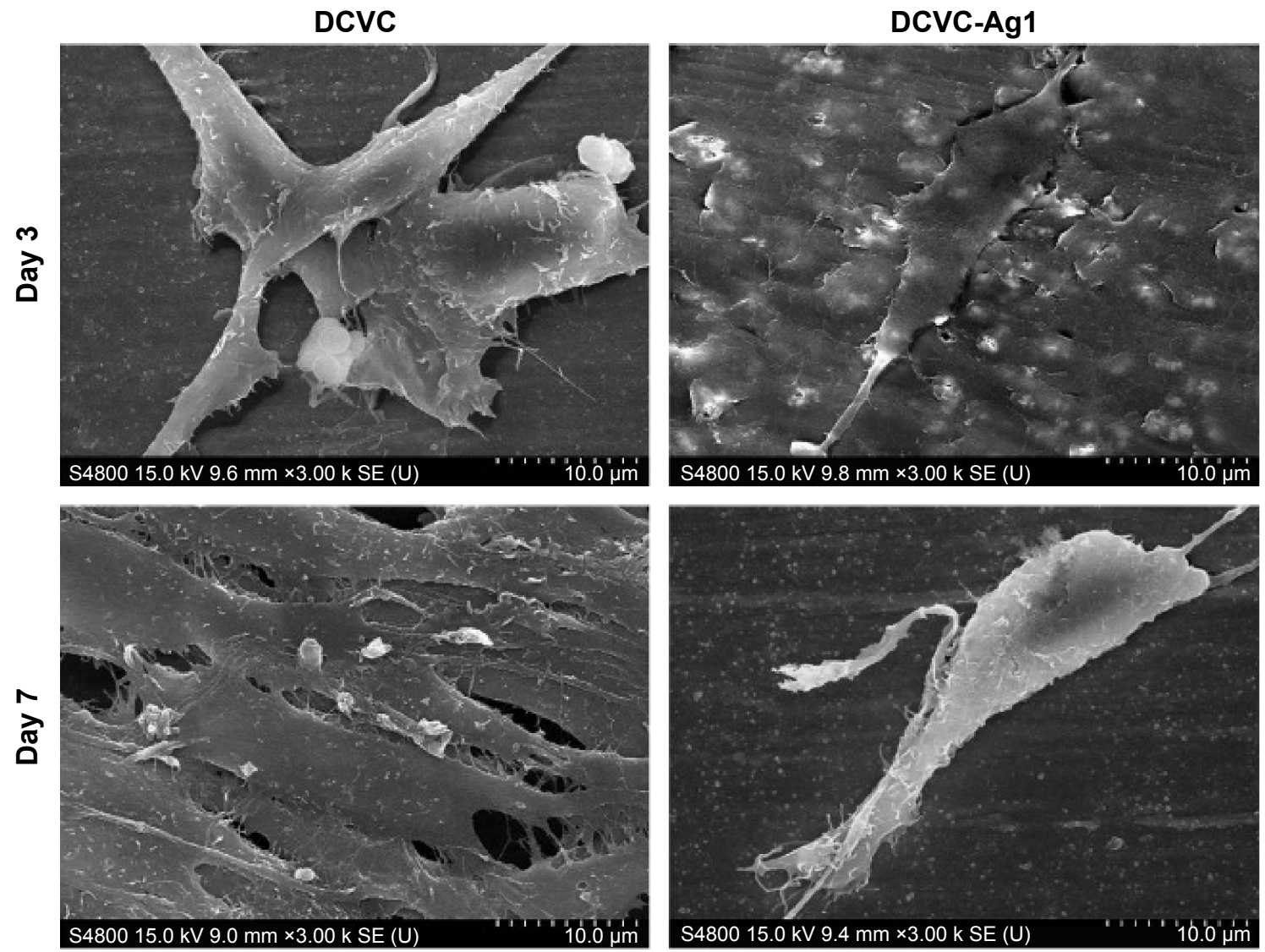

\section{DCVC-Ag2}
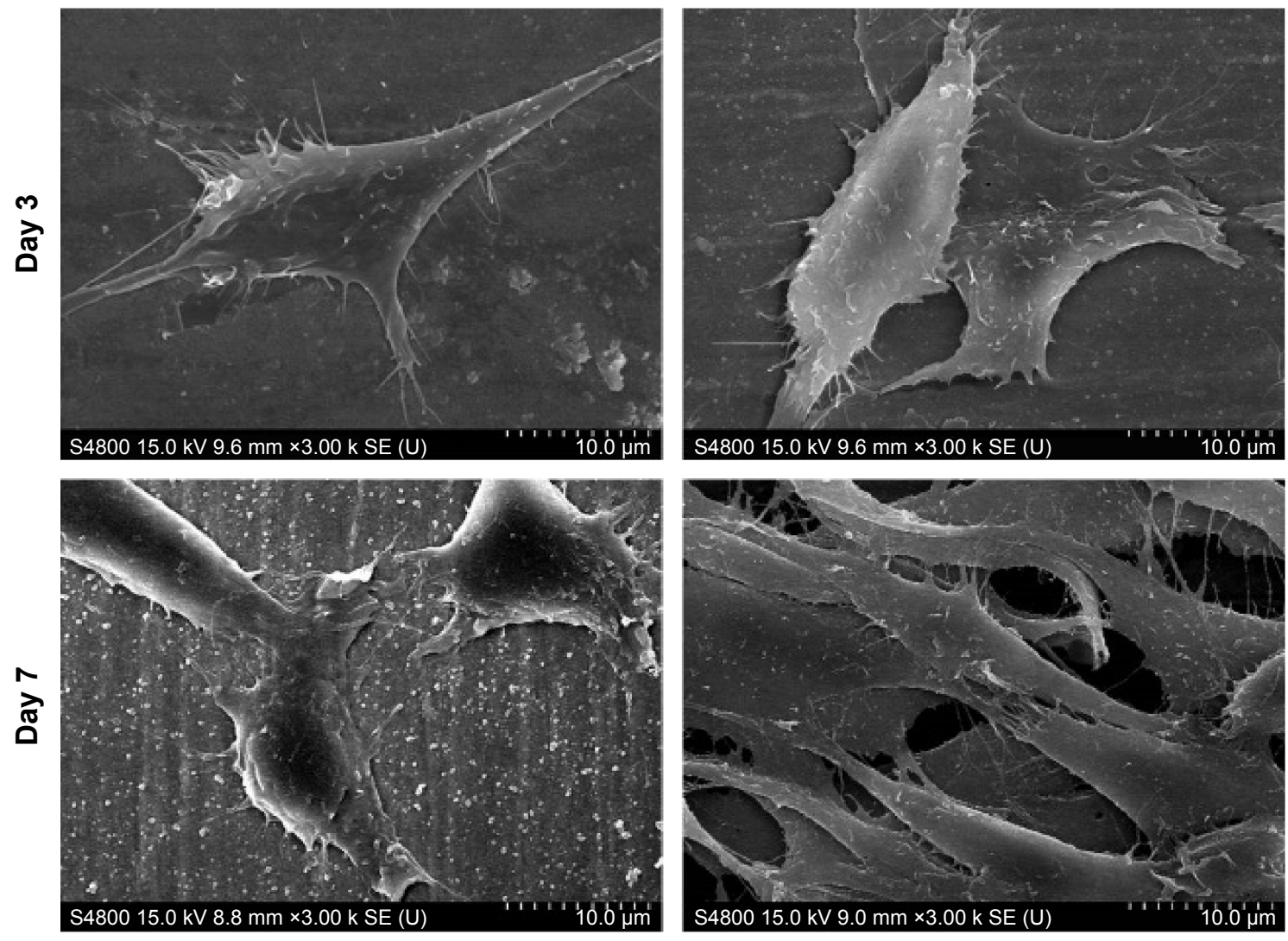

Figure 9 The SEM images of MC3T3-EI cell attachment on DCVC, DCVC-AgI, DCVC-Ag2, and DCVC-Ag3 samples for days 3 and 7. Abbreviations: DCVC, central venous catheters coated with polydopamine films; SEM, scanning electron microscopy. 
AgNPs density. The cytotoxicity was also associated with the content of coated AgNPs. The DCVC-Ag3 sample showed both obvious antibacterial ability and good biocompatibility. The results suggested that antibacterial and biocompatible AgNPs coating could be formed by carefully controlling the size and the content of nanoparticles, which has a promising prospect in clinical application.

\section{Acknowledgments}

The authors acknowledge financial support from the Young Foundation of the Affiliated Dongnan Hospital of Xiamen University (12Y001), Medical Scientific Youth Program Supported by Nanjing Command (18FBQN2014008) and National Natural Science Foundation of China (Grant No.51571169).

\section{Disclosure}

The authors report no conflicts of interest in this work.

\section{References}

1. Pongruangporn M, Ajenjo MC, Russo AJ, et al. Patient- and devicespecific risk factors for peripherally inserted central venous catheterrelated bloodstream infections. Infect Control Hosp Epidemiol. 2013; 34(2):184-189.

2. LeMaster CH, Schuur JD, Pandya D, et al. Infection and natural history of emergency department-placed central venous catheters. Annf Emerg Med. 2010;56(5):492-497.e491.

3. Sousa C, Henriques M, Oliveira R. Mini-review: antimicrobial central venous catheters - recent advances and strategies. Biofouling. 2011; 27(6):609-620.

4. Gilbert RE, Harden M. Effectiveness of impregnated central venous catheters for catheter related blood stream infection: a systematic review. Curr Opin Infect Dis. 2008;21(3):235-245.

5. Bong JJ, Kite P, Wilco MH, McMahon MJ. Prevention of catheter related bloodstream infection by silver iontophoretic central venous catheters: a randomised controlled trial. J Clin Pathol. 2003;56(10):731-735.

6. Knetsch ML, Koole LH. New strategies in the development of antimicrobial coatings: the example of increasing usage of silver and silver nanoparticles. Polymers. 2011;3(1):340-366.

7. Macocinschi D, Filip D, Paslaru E, et al. Polyurethane - extracellular matrix/silver bionanocomposites for urinary catheters. J Bioact Compat Polym: Biomed Appl. 2014:0883911514560661.

8. Filip D, Macocinschi D, Paslaru E, et al. Polyurethane biocompatible silver bionanocomposites for biomedical applications. J Nanopart Res. 2014;16(11): 17 .

9. Agarwala M, Barman T, Gogoi D, Choudhury B, Pal AR, Yadav RNS. Highly effective antibiofilm coating of silver-polymer nanocomposite on polymeric medical devices deposited by one step plasma process. J Biomed Mater Res. Part B. 2014;102(6):1223-1235.

10. Saez S, Fasciani C, Stamplecoskie KG, et al. Photochemical synthesis of biocompatible and antibacterial silver nanoparticles embedded within polyurethane polymers. Photochem Photobiol Sci. 2015;14(4): 661-664.

11. Lee H, Dellatore SM, Miller WM, Messersmith PB. Mussel-inspired surface chemistry for multifunctional coatings. Science. 2007;318(5849): 426-430.

12. Lee H, Lee BP, Messersmith PB. A reversible wet/dry adhesive inspired by mussels and geckos. Nature. 2007;448(7151):338-334.

13. Wang W, Jiang Y, Liao Y, Tian M, Zou H, Zhang L. Fabrication of silver-coated silica microspheres through mussel-inspired surface functionalization. J Colloid Interface Sci. 2011;358(2):567-574.
14. Wang W, Cheng W, Tian M, Zou H, Li L, Zhang L. Preparation of PET/Ag hybrid fibers via a biomimetic surface functionalization method. Electrochimica Acta. 2012;79:37-45.

15. Liao Y, Wang Y, Feng X, Wang W, Xu F, Zhang L. Antibacterial surfaces through dopamine functionalization and silver nanoparticle immobilization. Mater Chem Phys. 2010;121(3):534-540.

16. Sileika TS, Kim H-D, Maniak P, Messersmith PB. Antibacterial performance of polydopamine-modified polymer surfaces containing passive and active components. ACS Appl Mater Interfaces. 2011;3(12):4602-4610.

17. XuH, Shi X, MaH, Lv Y, Zhang L, MaoZ. The preparation and antibacterial effects of dopa-cotton/AgNPs. Appl Surf Sci. 2011;257(15):6799-6803.

18. Sureshkumar M, Lee P-N, Lee C-K. Stepwise assembly of multimetallic nanoparticles via self-polymerized polydopamine. J Mater Chem. 2011;21(33):12316-12320.

19. Tan KL, Woon LL, Wong HK, Kang ET, Neoh KG. Surface modification of plasma-pretreated poly(tetrafluoroethylene) films by graftcopolymerization. Macromolecules. 1993;26(11):2832-2836.

20. Lai Y, Zhuang H, Xie K, et al. Fabrication of uniform $\mathrm{Ag} / \mathrm{TiO} 2$ nanotube array structures with enhanced photoelectrochemical performance. New J Chem. 2010;34(7):1335-1340.

21. Li J, Qiao Y, Zhu H, Meng F, Liu X. Existence, release, and antibacterial actions of silver nanoparticles on $\mathrm{Ag}-\mathrm{PIII} \mathrm{TiO}_{2}$ films with different nanotopographies. Int J Nanomed. 2014;9:3389-3402.

22. Saito Y, Wang JJ, Smith DA, Batchelder DN. A simple chemical method for the preparation of silver surfaces for efficient SERS. Langmuir. 2002; 18(8):2959-2961.

23. Juan L, Zhimin Z, Anchun M, Lei L, Jingchao Z. Deposition of silver nanoparticles on titanium surface for antibacterial effect. Int J Nanomed. 2010;5:261-267.

24. Leuck A-M, Johnson JR, Hunt MA, et al. Safety and efficacy of a novel silver-impregnated urinary catheter system for preventing catheterassociated bacteriuria: a pilot randomized clinical trial. Am J Infect Control. 2015;43(3):260-265.

25. Lajcak M, Heidecke V, Haude KH, Rainov NG. Infection rates of external ventricular drains are reduced by the use of silver-impregnated catheters. Acta Neurochir. 2013;155(5):875-881.

26. Wang X, Cheng F, Gao J, Wang L. Antibacterial wound dressing from chitosan/polyethylene oxide nanofibers mats embedded with silver nanoparticles. J Biomater Appl. 2015;29(8):1086-1095.

27. Park EJ, Yi J, Kim Y, Choi K, Park K. Silver nanoparticles induce cytotoxicity by a trojan-horse type mechanism. Toxicol In Vitro. 2010; 24(3):872-878.

28. Zhao L, Wang H, Huo K, et al. Antibacterial nano-structured titania coating incorporated with silver nanoparticles. Biomaterials. 2011; 32(24):5706-5716.

29. Johnston HJ, Hutchison G, Christensen FM, Peters S, Hankin S, Stone V. A review of the in vivo and in vitro toxicity of silver and gold particulates: particle attributes and biological mechanisms responsible for the observed toxicity. Crit Rev Toxicol. 2010;40(4):328-346.

30. Albers CE, Hofstetter W, Siebenrock KA, Landmann R, Klenke FM. In vitro cytotoxicity of silver nanoparticles on osteoblasts and osteoclasts at antibacterial concentrations. Nanotoxicology. 2013;7(1):30-36.

31. Sureshkumar M, Siswanto DY, Chen Y-C, Lee C-K, Wang M-J. Antibacterial and biocompatible surfaces based on dopamine autooxidized silver nanoparticles. J Polym Sci B Polym Phys. 2013;51(4):303-310.

32. Politano AD, Campbell KT, Rosenberger LH, Sawyer RG. Use of silver in the prevention and treatment of infections: silver review. Surg Infect. 2013;14(1):8-20.

33. Zhao Y, Xing Q, Janjanam J, et al. Facile electrochemical synthesis of antimicrobial $\mathrm{TiO}_{2}$ nanotube arrays. Int J Nanomed. 2014;9:5177-5187.

34. Lee KJ, Nallathamby PD, Browning LM, Osgood CJ, Xu X-HN. In vivo imaging of transport and biocompatibility of single silver nanoparticles in early development of zebrafish embryos. Acs Nano. 2007;1(2): 133-143.

35. Travan A, Pelillo C, Donati I, et al. Non-cytotoxic silver nanoparticlepolysaccharide nanocomposites with antimicrobial activity. Biomacromolecules. 2009;10(6):1429-1435. 


\section{Publish your work in this journal}

The International Journal of Nanomedicine is an international, peerreviewed journal focusing on the application of nanotechnology in diagnostics, therapeutics, and drug delivery systems throughout the biomedical field. This journal is indexed on PubMed Central, MedLine, CAS, SciSearch $\AA$, Current Contents $\AA /$ Clinical Medicine,
Journal Citation Reports/Science Edition, EMBase, Scopus and the Elsevier Bibliographic databases. The manuscript management system is completely online and includes a very quick and fair peer-review system, which is all easy to use. Visit http://www.dovepress.com/ testimonials.php to read real quotes from published authors.

Submit your manuscript here: http://www.dovepress.com/international-journal-of-nanomedicine-journal 